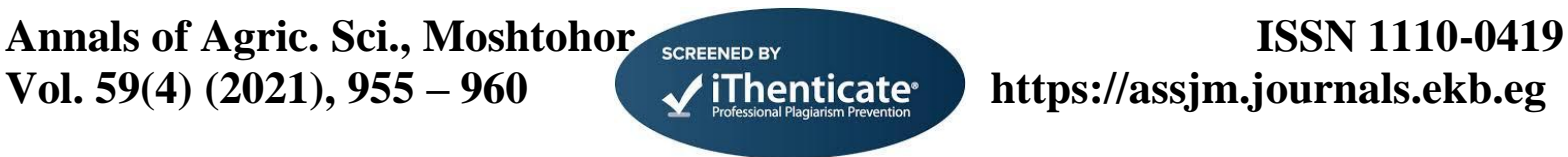

\title{
Response of Washington Navel Orange Trees to Foliar Spray with Some Stimulents
}

\author{
2- $\quad$ YIELD INDICATORS AND FRUIT QUALITY
}

El Wakeel, M. K., Atawia, A. R.; Abd Ellatif, F. M. and Bakry, Kh. A.

\begin{abstract}
The present study carried out on fruitful Washington Navel orange trees 12 years old, budded on sour orange rootstock grown in clay loamy soils in private farm at Toukh region, El-Kalubeia Governorate to evaluate response the trees productivity to foliar spray with some stimulating compounds at two concentrations for each i.e., yeast extract at $2.50 \mathrm{ml} / 1 \& 5.0 \mathrm{ml} / 1$, sea weeds at $0.2 \& 0.4 \mathrm{ml} / \mathrm{l}$, promalin at $100 \& 200 \mathrm{ppm}$ and humic acid at $3.0 \& 6.0 \mathrm{~g} / \mathrm{l}$ and mixture of lower concentration of each substance, beside control which sprayed with tap water during 2019 and 2020 seasons. The results indicated that, treatments significantly increased yield indicators and improved fruit quality compared to control. Promalin at $200 \mathrm{ppm}$ or yeast extract at $2.5 \mathrm{~m} / \mathrm{L}+\mathrm{sea}$ weeds extract at $0.2 \mathrm{~m} / \mathrm{L}+$ promalin at $100 \mathrm{ppm}$ gave highest values of yield measurements and fruit quality.
\end{abstract}

Key words: yeast extract, sea weeds extract, promalin, humic acid, citrus, yield, fruit quality.

\section{Introduction}

The citrus (Citrus spp.) is considered one of the most important fruit crops grown in many tropical and subtropical countries. The Egyptian Agriculture statistics in 2019 indicate that, citrus total planted area reached 477510 feddans and total citrus production 420000 tons of fruits, this represents $28.78 \%$ and $37.08 \%$ of total fruits orchards area and fruits production, respectively. Total orange cultivated area represents 326484 feddans with total fruits production of 3147545 tons. Ministry of Agriculture, Economic Affairs Sector, 2019.

Yeast extract, sea weeds extract and humic acid is a new generation of natural organic fertilizers containing highly effective nutritious and increase yield. Unlike, chemical fertilizers, extracts derived from sea-weeds are biodegradable, nontoxic, nonpolluting and non-hazardous to human, animals and birds (Dhargalkar and Pereira, 2005).

Yeast extract and sea weeds extract are found to be viable alternatives to fertilizing input for agricultural crops due to its high level of organic matter, micro and macro elements, vitamins, fatty acids, also rich in growth regulators (Crouch and Van-Staden, 1993).

Yeast as a natural source of cytokinins stimulates cell division and enlargement as well as the synthesis of protein, nucleic acid and chlorophyll (Atawia and El-Desouky, 1998).

Yeast is one of the richest source of high quality protein, namely the essential amino acids like lysine, tryptophan etc., contains the essential minerals and trace elements, namely calcium, cobalt, iron etc. and the best sources of the B-complex vitamins such as B1, B2, B6 and B12. The extract is a valuable source of bio-constituents especially, cytokinins (Amer, 2004).

Sea weeds extract application for different crops has a great importance due to its content with high levels of organic matter, micro elements, vitamins and amino acids and also, is being rich in growth regulators (Khan et al., 2009)

The number and weight of fruits /tree and fruit TSS, TSS/Acid ratio and peel thickness were significantly increased when Washington Navel orange trees were sprayed with promalin $(\mathrm{BA}+\mathrm{GA}$ 4+7) (Ibrahim et al., 1994). Spray apple trees with promalin just after full bloom, and the second application one week later increased fruit size and length (Burak et al., 1998).

Vaughan et al., (1985) showed that, humic substances increased yield by acting on mechanisms involved in: cell respiration, photosynthesis, protein synthesis, water, and nutrient uptake, enzyme activities.

Hence, this study aimed to increase yield and improve fruit quality of Washington Navel orange trees by using some stimulating substances i.e., yeast extract, sea weeds extract, promalin and humic acid.

\section{Materials and Methods}

The present studywas carried out in private farm at Toukh region, El-Kalubeia Governorate during two successive seasons 2019 and 2020 seasons to study the effect of some stimulating compounds on fruitful Washington Navel orange trees (Citrus sinensis) budded on sour orange rootstock. The trees were 12 year-old, grown in clay loamy soil, planted at $5 \times 5$ meters and subjected to the recommended management of orange orchard. Thirty trees of almost similar as possible in growth vigour, free from diseases were carefully selected and divided into ten treatments each included three replicates. Each replicate was represented by a single tree.

The Washington Navel orange (Citrus sinensis) trees were sprayed monthly from first March to first July (five times) with stimulating compounds with 
two concentrations from each (yeast extract at 2.5 $\mathrm{ml} / \mathrm{l} \& 5.0 \mathrm{ml} / \mathrm{l}$, sea weeds extract at $0.2 \& 0.4 \mathrm{ml} / \mathrm{l}$, promalin at $100 \& 200 \mathrm{ppm}$ and humic acid at 3.0 $\& 6.0 \mathrm{~g} / \mathrm{l})$ and mixture of first concentration of each compound in addition to the control treatment which was sprayed with tap water only during two experimental 2019 and 2020 seasons.

Each tree was sprayed with 5 liters of spraying solution or water (control) using hand sprayer to the run off. The applied treatments could be summarized as follows:

- Control (trees sprayed with tap water). -Yeast extract at 2.5 and $5.0 \mathrm{~m} / \mathrm{l}$. - Sea-weed extract at 0.2 and $0.4 \mathrm{~m} / 1$. -promalin at 100 and $200 \mathrm{ppm}$. -humic acid at 3.0 and $6.0 \mathrm{~g} / 1$. - yeast extract at $2.5 \mathrm{ml} / \mathrm{l}$. + sea-weed extract at $0.2 \mathrm{~m} / \mathrm{l}$. + promalin at $100 \mathrm{ppm}$. + humic acid at $3.0 \mathrm{~g} / \mathrm{l}$.

\section{- Experiment lay out:}

The complete randomized block design with three replicates was employed for arranging the ten investigated treatments, (including control) whereas each replicate was represented by a single tree. Consequently, thirty healthy fruitful Washington Navel orange trees were carefully selected, as being healthy, disease free. Chosen trees were divided according to their growth vigour into four categories (blocks) each included ten similar trees for receiving the investigated nine stimulating treatments besides the control (tap water spray).

Taking into consideration that, spray treatments were applied covering the whole foliage of each tree canopy, whereas 5 liters found to be sufficient in this concern.

Methodology as has been reported in this study in order to evaluate the response of Washington Navel orange trees under studied to various stimulating treatments were carried out through determining changes in different measurements of the following examined characteristics:

\section{- Fruiting measurements:}

- Fruit set percentage:

At full bloom during each experimental season number of perfect flowers per each tagged limb was counted. After $75 \%$ of petal fall fruit set as percentage of perfect flowers were estimated according to the following equation described by Westwood (1978).

$$
100 \times \frac{\text { Number of set fruitlets }}{\text { Number of perfect flowers }}=\% \text { set Fruit }
$$

- Fruits drop and retention\%:

Percentage of both dropped and retained fruits, were determined on $25^{\text {th }}$ June according to the following equations:

$$
100 \times \frac{\text { Number of dropped fruits }}{\text { Number of set fruitlets }}=\% \text { drop Fruit }
$$

$$
100 \times \frac{\text { Number of remained fruits }}{\% \text { retained }}
$$

Fruit

- Yield:

At first week of December 2019 and 2020 fruits of each individual tree were separately harvested, then counted and weighed. Tree productivity (yield) was estimated either as number and weight $(\mathrm{kg})$ of harvested fruits per each tree.

-Fruit quality:

- Fruit physical characteristics:

In this regard average fruit weight (g.); volume (ml); dimensions (polar \& equatorial diameters i.e., length \& width in $\mathrm{cm}$.); fruit shape index (length: width); peel/rind thickness ( $\mathrm{mm}$ ) and juice volume (cc) were the fruit physical characteristics investigated in this regard.

\section{-Fruit chemical properties:}

Fruit juice total soluble solids percentage (TSS\%) was determined using Carl Zeis hand refractometer. Total acidity as g. of unhydrous citric acid per $100 \mathrm{ml}$ fruit juice was determined by A.O.A.C., (2000). Total soluble solids/ acid ratio were also estimated. Ascorbic, acid (Vitamin C) content was determined using 2,6 dichlorophenol indophenol indicator for titration according to A.O.A.C., (2000). Moreover, total sugars\% was determined after the method described by Smith et al., (1956).

\section{- Statistical analysis:}

All data obtained data during the two seasons were statistically analyzed using the analysis of variance method according to Sendecor and Cochran (1990). However, means were distinguished by the Duncan's Multiple Range Test (Duncan, 1955), which used to compare between them.

\section{Results and Discussion}

Data obtained during both 2019 and 2020 experimental seasons could be summarized as follow:

-Yield indicators:

Data in Tables (1 and 2) showed that, the effect of different stimulating compounds i.e. yeast extract at $2.5 \& 5.0 \mathrm{ml} / 1$; sea weeds extract at $0.2 \& 0.4 \mathrm{~m} / 1$; promalin at $100 \& 200 \mathrm{ppm}$; humic acid at $3.0 \& 6.0$ $\mathrm{g} / \mathrm{l}$ and yeast extract at $2.5 \mathrm{~m} / \mathrm{l}+$ sea weeds extract at $0.2 \mathrm{~m} / 1+$ promalin at $100 \mathrm{ppm}$ on yield indicators i.e., fruit set $\%$, fruit drop $\%$, fruit retention $\%$, number of fruits/ tree and weight of fruits per tree $(\mathrm{kg})$ of Washington navel orange trees in both experimental seasons. All applied treatments significantly increased fruit set $\%$, fruit retention $\%$, number of fruits/ tree and weight of fruits per tree $(\mathrm{kg})$ over the control. Fruit drop percentage gave low value during two seasons of study. The maximum fruit set\%, fruit retention\%, number of fruits/ tree and weight 
of fruits per tree $(\mathrm{kg})$ with using promalin at 200 ppm during 2019 and 2020 seasons. Promalin at $200 \mathrm{ppm}$ or yeast extract at $2.5 \mathrm{~m} / \mathrm{l}+$ sea weeds extract at $0.2 \mathrm{~m} / 1+$ promalin at $100 \mathrm{ppm}$ gave second significantly increased, promalin at 100 came third significantly increased in fruit set\%, fruit retention \%, number of fruits/ tree and weight of fruits per tree $(\mathrm{kg})$ during the first and second seasons. In addition humic acid at 3.0 and $6.0 \mathrm{~g} / \mathrm{l}$ gave lowest significantly increased as compared with the control in both seasons.

Generally concluded that, different stimulating (promalin, sea weeds extract, humic acid and yeast extract concentrations) significantly increased fruit set\%, fruit retention\%, number of fruits/ tree and weight of fruits per tree $(\mathrm{kg})$ of Washington Navel orange trees. However, such increment was superior for promalin at $200 \mathrm{ppm}$ and lower in case of humic acid at $6 \mathrm{~g} / \mathrm{l}$ in the two seasons.

In this respect, the increase in fruit set $\%$ resulted from different stimulating compared, may be attributed to reduction in flowers abscission \% which in turn reflected to enhancement of fruit setting. In addition such treatments may be stimulates the synthesis of total carbohydrates, protein and mineral concentrations in the leaves (source) as well as the endogenous auxins, especially at full blooming and setting stages there by increased fruit setting percentages. The obtained results regarding the increment of fruit set\% exhibited by different biostimulating compounds goes in the line with those found by El-Shazly and Mustafa (2013) in Washington Navel orange trees.

Table 1. Effect of foliar spray with yeast extract, sea weeds extract, promalin and humic acid on fruit set, fruit drop and fruit retention percentages of Washington Navel orange trees during 2019 and 2020 seasons.

\begin{tabular}{|c|c|c|c|c|c|c|}
\hline \multirow{2}{*}{$\begin{array}{l}\text { Parameters } \\
\text { Treatments }\end{array}$} & \multicolumn{2}{|c|}{ Fruit set( \%) } & \multicolumn{2}{|c|}{ Fruit drop (\%) } & \multicolumn{2}{|c|}{ Fruit retention $(\%)$} \\
\hline & 2019 & 2020 & 2019 & 2020 & 2019 & 2020 \\
\hline T1- Control & $14.00 \mathrm{~h}$ & $14.14 \mathrm{~h}$ & $90.80 \mathrm{a}$ & $90.70 \mathrm{a}$ & $9.20 \mathrm{~d}$ & $9.30 \mathrm{e}$ \\
\hline T2- Yeast extract at $2.5 \mathrm{~m} / \mathrm{l}$ & $19.67 \mathrm{~d}$ & $20.74 \mathrm{~d}$ & $86.26 \mathrm{c}$ & $85.23 \mathrm{~d}$ & $13.74 \mathrm{~b}$ & $14.77 \mathrm{~b}$ \\
\hline T3-Yeast extract at $5.0 \mathrm{~m} / \mathrm{l}$ & $21.10 \mathrm{c}$ & $22.37 \mathrm{c}$ & $85.20 \mathrm{c}$ & $84.13 \mathrm{~d}$ & $14.80 \mathrm{~b}$ & $15.87 \mathrm{~b}$ \\
\hline T4- Sea weeds extract at $0.2 \mathrm{~m} / \mathrm{l}$ & $15.77 \mathrm{f}$ & $16.54 \mathrm{f}$ & $90.46 \mathrm{a}$ & $89.90 \mathrm{ab}$ & $9.54 \mathrm{~d}$ & $10.10 \mathrm{de}$ \\
\hline T5- Sea weeds extract at $0.4 \mathrm{~m} / 1$ & $17.14 \mathrm{e}$ & $18.77 \mathrm{e}$ & $88.16 \mathrm{~b}$ & $87.26 \mathrm{c}$ & $11.84 \mathrm{c}$ & $12.74 \mathrm{c}$ \\
\hline T6- Promalin at 100 ppm & $22.04 \mathrm{~b}$ & $23.74 \mathrm{~b}$ & $83.23 \mathrm{~d}$ & $82.04 \mathrm{e}$ & $16.77 \mathrm{a}$ & $17.97 \mathrm{a}$ \\
\hline T7- Promalin at $200 \mathrm{ppm}$ & $23.14 \mathrm{a}$ & $25.14 \mathrm{a}$ & $82.10 \mathrm{~d}$ & $81.00 \mathrm{e}$ & $17.90 \mathrm{a}$ & $19.00 \mathrm{a}$ \\
\hline T8- Humic acid at $3.0 \mathrm{~g} / \mathrm{l}$ & $17.64 \mathrm{e}$ & $19.27 \mathrm{e}$ & $89.73 \mathrm{ab}$ & $89.00 \mathrm{~b}$ & $10.27 \mathrm{~cd}$ & $11.00 \mathrm{~d}$ \\
\hline T9- Humic acid at $6.0 \mathrm{~g} / \mathrm{l}$ & $14.64 \mathrm{~g}$ & $15.54 \mathrm{~g}$ & $90.73 \mathrm{a}$ & $90.26 \mathrm{ab}$ & $9.27 \mathrm{~d}$ & $9.74 \mathrm{de}$ \\
\hline $\mathrm{T} 10=\mathrm{T} 2+\mathrm{T} 4+\mathrm{T} 6+\mathrm{T} 8$ & $22.07 \mathrm{~b}$ & $23.77 \mathrm{~b}$ & $83.20 \mathrm{~d}$ & $82.00 \mathrm{e}$ & $16.80 \mathrm{a}$ & $18.00 \mathrm{a}$ \\
\hline
\end{tabular}

Mean followed by the same letters within each column are not significantly different at 0.5 level.

Table 2 . Effect of foliar spray with yeast extract, sea weeds extract, promalin and humic acid on number of fruits/tree and Yield (kg/tree) of Washington Navel

\begin{tabular}{lllll}
\hline \multirow{2}{*}{ Parameters } & \multicolumn{2}{l}{ Number of fruits/tree } & \multicolumn{2}{l}{ Yield $(\mathrm{kg} /$ tree $)$} \\
\cline { 2 - 5 } Treatments & 2019 & 2020 & 2019 & 2020 \\
\hline T1- Control & $217.67 \mathrm{f}$ & $219.33 \mathrm{f}$ & $44.43 \mathrm{~h}$ & $45.93 \mathrm{~h}$ \\
T2- Yeast extract at $2.5 \mathrm{~m} / 1$ & $257.67 \mathrm{~b}$ & $261.33 \mathrm{~b}$ & $69.96 \mathrm{c}$ & $72.01 \mathrm{c}$ \\
T3-Yeast extract at $5.0 \mathrm{~m} / 1$ & $260.00 \mathrm{a} \mathrm{b}$ & $264.33 \mathrm{ab}$ & $72.89 \mathrm{~b}$ & $75.26 \mathrm{~b}$ \\
T4- Sea weeds extract at $0.2 \mathrm{~m} / 1$ & $236.33 \mathrm{de}$ & $239.33 \mathrm{de}$ & $55.32 \mathrm{f}$ & $57.23 \mathrm{f}$ \\
T5- Sea weeds extract at $0.4 \mathrm{~m} / 1$ & $245.67 \mathrm{c}$ & $249.33 \mathrm{c}$ & $65.04 \mathrm{~d}$ & $67.04 \mathrm{~d}$ \\
T6- Promalin at $100 \mathrm{ppm}$ & $263.67 \mathrm{ab}$ & $268.00 \mathrm{ab}$ & $75.55 \mathrm{a}$ & $77.50 \mathrm{a}$ \\
T7- Promalin at $200 \mathrm{ppm}$ & $266.33 \mathrm{a}$ & $270.00 \mathrm{a}$ & $77.16 \mathrm{a}$ & $79.38 \mathrm{a}$ \\
T8- Humic acid at $3.0 \mathrm{~g} / 1$ & $241.33 \mathrm{~cd}$ & $244.33 \mathrm{~cd}$ & $61.36 \mathrm{e}$ & $63.13 \mathrm{e}$ \\
T9- Humic acid at $6.0 \mathrm{~g} / \mathrm{l}$ & $231.00 \mathrm{e}$ & $234.33 \mathrm{e}$ & $50.70 \mathrm{~g}$ & $52.02 \mathrm{~g}$ \\
T10= T2 + T4 + T6 + T8 & $264.00 \mathrm{ab}$ & $268.33 \mathrm{a}$ & $75.66 \mathrm{a}$ & $77.60 \mathrm{a}$ \\
\hline
\end{tabular}

Orange trees during 2019 and 2020 seasons.

Mean followed by the same letters within each column are not significantly different at 0.5 level.

- Fruit quality:

- Fruit physical characteristics:

In this regards, average fruit weight $(\mathrm{g})$, fruit volume $(\mathrm{ml})$, fruit dimensions (equatorial \& polar diameters), fruit shape index (L/D), fruit peel thickness $(\mathrm{mm})$ and fruit juice volume $(\mathrm{ml})$ were evaluated fruit physical characteristics of Washington Navel orange in response to different applied treatments.
Results in Tables (3, 4 and 5) showed that, different treatments i.e., yeast extract, sea weeds extract, promalin and humic acid significantly increased average fruit weight $(\mathrm{g})$, fruit volume (ml), fruit dimensions (equatorial \& polar diameters), fruit shape index (L/D), fruit peel thickness $(\mathrm{mm})$ and fruit juice volume $(\mathrm{ml})$ during two seasons. The maximum effect of these measurements with using promalin at $200 \mathrm{ppm}$. 
Second treatments increased with promalin at $200 \mathrm{ppm}$ or yeast extract at $2.5 \mathrm{~m} / \mathrm{l}+$ sea weeds extract at $0.2 \mathrm{~m} / 1+$ promalin at $100 \mathrm{ppm}+$ humic $3.0 \mathrm{~g} / \mathrm{l}$. Third significantly increased treatments with using promalin at $100 \mathrm{ppm}$ during both seasons. Least treatments increased compared with the control with using humic acid at two levels during both seasons of study.

Obtained results regarding the positive effects of bio-stimulating compounds on some fruit physical characteristics goes in the line of several investigation findings El-Shazly and Mustafa (2013) and Mangan and Thomas (2014) on some fruit physical properties.

Table 3. Effect of foliar spray with yeast extract, sea weeds extract, promalin and humic acid on fruit weight and volume of Washington Navel orange trees during 2019 and 2020 seasons.

\begin{tabular}{lllll}
\hline Parameters & \multicolumn{2}{c}{ Fruit weight $\mathbf{( g )}$} & \multicolumn{2}{l}{ Fruit volume (ml) } \\
\cline { 2 - 5 } Treatments & $\mathbf{2 0 1 9}$ & $\mathbf{2 0 2 0}$ & $\mathbf{2 0 1 9}$ & $\mathbf{2 0 2 0}$ \\
\hline T1- Control & $204.10 \mathrm{i}$ & $209.43 \mathrm{i}$ & $221.33 \mathrm{i}$ & $223.90 \mathrm{i}$ \\
T2- Yeast extract at $\mathbf{2 . 5} \mathbf{~ m} / \mathbf{L}$ & $271.50 \mathrm{~d}$ & $275.57 \mathrm{~d}$ & $274.13 \mathrm{~d}$ & $279.53 \mathrm{~d}$ \\
T3-Yeast extract at $\mathbf{5 . 0} \mathbf{~ m} / \mathbf{L}$ & $280.33 \mathrm{c}$ & $284.73 \mathrm{c}$ & $281.00 \mathrm{c}$ & $286.20 \mathrm{c}$ \\
T4- Sea weeds extract at $\mathbf{0 . 2 m} / \mathbf{L}$ & $234.07 \mathrm{~g}$ & $239.10 \mathrm{~g}$ & $241.83 \mathrm{~g}$ & $246.53 \mathrm{~g}$ \\
T5- Sea weeds extract at $\mathbf{0 . 4 m} / \mathbf{L}$ & $264.73 \mathrm{e}$ & $268.87 \mathrm{e}$ & $260.63 \mathrm{e}$ & $265.47 \mathrm{e}$ \\
T6- Promalin at $\mathbf{1 0 0} \mathbf{~ p p m}$ & $286.53 \mathrm{~b}$ & $289.17 \mathrm{~b}$ & $288.60 \mathrm{~b}$ & $293.67 \mathrm{~b}$ \\
T7- Promalin at $\mathbf{2 0 0} \mathbf{~ p p m}$ & $289.73 \mathrm{a}$ & $294.00 \mathrm{a}$ & $293.03 \mathrm{a}$ & $298.93 \mathrm{a}$ \\
T8- Humic acid at 3.0 g/L & $254.23 \mathrm{f}$ & $258.37 \mathrm{f}$ & $251.10 \mathrm{f}$ & $257.67 \mathrm{f}$ \\
T9- Humic acid at $\mathbf{6 . 0} \mathbf{~ g / L ~}$ & $219.47 \mathrm{~h}$ & $222.00 \mathrm{~h}$ & $232.03 \mathrm{~h}$ & $236.17 \mathrm{~h}$ \\
T10= T2 + T4 + T6+ T8 & $286.57 \mathrm{~b}$ & $289.20 \mathrm{~b}$ & $288.63 \mathrm{~b}$ & $293.70 \mathrm{~b}$ \\
\hline
\end{tabular}

Mean followed by the same letters within each column are not significantly different $r$ at 0.5 level.

Table 4. Effect of foliar spray with yeast extract, sea weeds extract, promalin and humic acid on fruit length, diameter and shape index of Washington Navel orange fruits during 2019 and 2020 seasons.

\begin{tabular}{|c|c|c|c|c|c|c|}
\hline \multirow[t]{2}{*}{$\begin{array}{l}\text { Parameters } \\
\text { Treatments }\end{array}$} & \multicolumn{2}{|c|}{$\begin{array}{l}\text { Fruit length } \\
\text { (mm) }\end{array}$} & \multicolumn{2}{|c|}{$\begin{array}{l}\text { Fruit diameter } \\
(\mathbf{m m})\end{array}$} & \multicolumn{2}{|c|}{$\begin{array}{l}\text { Fruit shape index } \\
\text { (L/D) }\end{array}$} \\
\hline & 2019 & 2020 & 2019 & 2020 & 2019 & 2020 \\
\hline T1- Control & $69.60 \mathrm{~g}$ & $70.40 \mathrm{~h}$ & $66.54 \mathrm{~g}$ & $67.90 \mathrm{i}$ & $1.04 \mathrm{~cd}$ & $1.03 \mathrm{~d}$ \\
\hline T2- Yeast extract at $2.5 \mathrm{~m} / \mathrm{l}$ & $79.37 \mathrm{~d}$ & $80.54 \mathrm{~d}$ & $73.30 \mathrm{~cd}$ & $76.34 \mathrm{~d}$ & $1.08 \mathrm{~b}$ & $1.05 \mathrm{c}$ \\
\hline T3-Yeast extract at $5.0 \mathrm{~m} / \mathrm{l}$ & $81.40 \mathrm{c}$ & $83.94 \mathrm{c}$ & $76.77 \mathrm{~b}$ & $78.40 \mathrm{c}$ & $1.06 \mathrm{bc}$ & $1.07 \mathrm{~b}$ \\
\hline $\begin{array}{l}\text { T4- Sea weeds extract at } \\
0.2 \mathrm{~m} / \mathrm{l}\end{array}$ & $70.44 \mathrm{~g}$ & $71.47 \mathrm{~g}$ & 69.17 ef & $70.47 \mathrm{~g}$ & $1.01 \mathrm{de}$ & $1.01 \mathrm{e}$ \\
\hline $\begin{array}{l}\text { T5- Sea weeds extract at } \\
0.4 \mathrm{~m} / \mathrm{l}\end{array}$ & $76.90 \mathrm{e}$ & $78.57 \mathrm{e}$ & $74.10 \mathrm{c}$ & $75.54 \mathrm{e}$ & 1.03 cde & $1.04 \mathrm{~d}$ \\
\hline T6- Promalin at 100 ppm & $84.14 b$ & $86.37 \mathrm{~b}$ & $75.50 \mathrm{bc}$ & $79.24 \mathrm{~b}$ & $1.11 \mathrm{a}$ & $1.09 \mathrm{a}$ \\
\hline T7- Promalin at $200 \mathrm{ppm}$ & $86.30 \mathrm{a}$ & $88.54 \mathrm{a}$ & $79.84 \mathrm{a}$ & $80.54 \mathrm{a}$ & $1.08 \mathrm{~b}$ & $1.10 \mathrm{a}$ \\
\hline T8- Humic acid at $3.0 \mathrm{~g} / \mathrm{l}$ & $72.17 \mathrm{f}$ & $74.77 \mathrm{f}$ & $71.04 \mathrm{de}$ & $73.90 \mathrm{f}$ & $1.01 \mathrm{e}$ & $1.01 \mathrm{e}$ \\
\hline T9- Humic acid at $6.0 \mathrm{~g} /$ & $70.37 \mathrm{~g}$ & $70.64 \mathrm{~h}$ & $67.37 \mathrm{fg}$ & $68.47 \mathrm{~h}$ & $1.04 \mathrm{cde}$ & $1.03 \mathrm{~d}$ \\
\hline $\mathrm{T} 10=\mathrm{T} 2+\mathrm{T} 4+\mathrm{T} 6+\mathrm{T} 8$ & $84.20 \mathrm{~b}$ & $86.44 \mathrm{~b}$ & $75.57 \mathrm{bc}$ & $79.30 \mathrm{~b}$ & $1.11 \mathrm{a}$ & $1.09 \mathrm{a}$ \\
\hline
\end{tabular}

Mean followed by the same letters within each column are not significantly different at 0.5 level.

Table 5. Effect of foliar spray with yeast extract, sea weeds extract, promalin and humic acid on Fruit peel thickness and Juice volume of Washington Navel orange fruits during 2019 and 2020 seasons.

\begin{tabular}{lllll}
\hline Parameters & \multicolumn{2}{c}{ Fruit peel thickness $\mathbf{( m m})$} & \multicolumn{2}{l}{ Juice volume $(\mathbf{m l})$} \\
\cline { 2 - 5 } Treatments & $\mathbf{2 0 1 9}$ & $\mathbf{2 0 2 0}$ & $\mathbf{2 0 1 9}$ & $\mathbf{2 0 2 0}$ \\
\hline T1- Control & $2.48 \mathrm{f}$ & $2.51 \mathrm{e}$ & $71.74 \mathrm{i}$ & $74.43 \mathrm{~h}$ \\
T2- Yeast extract at $\mathbf{2 . 5} \mathbf{~ m} / \mathbf{l}$ & $2.86 \mathrm{~cd}$ & $2.90 \mathrm{c}$ & $92.17 \mathrm{~d}$ & $95.43 \mathrm{c}$ \\
T3-Yeast extract at $\mathbf{5 . 0 ~} \mathbf{~} / \mathbf{l}$ & $2.99 \mathrm{bc}$ & $3.05 \mathrm{~b}$ & $94.37 \mathrm{c}$ & $98.63 \mathrm{~b}$ \\
T4- Sea weeds extract at $\mathbf{0 . 2 m} / \mathbf{l}$ & $2.56 \mathrm{ef}$ & $2.61 \mathrm{de}$ & $80.57 \mathrm{~g}$ & $84.33 \mathrm{f}$ \\
T5- Sea weeds extract at $\mathbf{0 . 4 m} / \mathbf{l}$ & $2.79 \mathrm{~d}$ & $2.82 \mathrm{c}$ & $87.67 \mathrm{e}$ & $90.70 \mathrm{~d}$ \\
T6- Promalin at $\mathbf{1 0 0} \mathbf{~ p p m}$ & $3.04 \mathrm{~b}$ & $3.17 \mathrm{a}$ & $97.50 \mathrm{~b}$ & $101.63 \mathrm{a}$ \\
T7- Promalin at $\mathbf{2 0 0} \mathbf{~ p p m}$ & $3.20 \mathrm{a}$ & $3.26 \mathrm{a}$ & $99.34 \mathrm{a}$ & $103.00 \mathrm{a}$ \\
T8- Humic acid at $\mathbf{3 . 0} \mathbf{~ g / l}$ & $2.64 \mathrm{e}$ & $2.69 \mathrm{~d}$ & $83.77 \mathrm{f}$ & $87.47 \mathrm{e}$ \\
T9- Humic acid at $\mathbf{6 . 0} \mathbf{~ g / l}$ & $2,52 \mathrm{ef}$ & $2.55 \mathrm{e}$ & $76.50 \mathrm{~h}$ & $79.40 \mathrm{~g}$ \\
T10= T2 + T4 + T6+ T8 & $3.10 \mathrm{ab}$ & $3.21 \mathrm{a}$ & $97.54 \mathrm{~b}$ & $101.67 \mathrm{a}$ \\
\hline
\end{tabular}

Mean followed by the same letters within each column are not significantly different at 0.5 level. 
- Fruit chemical properties:

In this regards fruit juice total soluble solids (TSS\%), total acidity, TSS /acid ratio, total sugars and vitamin $\mathrm{C}$ contents were different investigated fruit juice chemical properties for Washington Navel orange regarding their response to the evaluated (yeast extract, sea weeds extract, promalin and humic acid) treatments.

Data tabulated in Tables (6 and 7) showed that, different applied treatments were significantly increased fruit juice total soluble solids (TSS \%), TSS /acid ratio, total sugars and vitamin C contents of Washington Navel orange fruits during both seasons. The superior treatment of fruit juice total soluble solids (TSS) \%, TSS /acid ratio, total sugars and ascorbic acid (vitamin C) contents with using promalin at $200 \mathrm{ppm}$. Second superior treatments with using yeast extract at $2.5 \mathrm{~m} / \mathrm{L}+$ sea weeds extract at $0.2 \mathrm{ml} / 1+$ promalin at $100 \mathrm{ppm}+$ humic acid at $3.0 \mathrm{~g} / \mathrm{L}$ during two seasons. Promalin at 100 ppm gave the third increase of fruit juice total soluble solids (TSS) \%, TSS /acid ratio, total sugars and ascorbic acid (vitamin C) contents during two seasons. Humic acid at two concentrations gave low significant increase in fruit juice total soluble solids (TSS) \%, TSS /acid ratio, total sugars and ascorbic acid (vitamin C) contents as compared with the control during both experimental seasons. Concerning fruit juice total acidity $\%$ contents data indicated that, different applied treatments i.e. yeast extract at $2.5 \& 5.0 \mathrm{~m} / \mathrm{L}$, sea weeds extract at $0.2 \&$ $0.4 \mathrm{ml} / 1$, promalin at $100 \& 200 \mathrm{ppm}$ and humic acid at $3.0 \& 6.0 \mathrm{~g} / \mathrm{L}$ were significantly reduced total acidity $\%$ in fruits of Washington Navel orange during both seasons of study.

The present results goes partially in line with that pointed out by several investigators regarding the beneficial effects of different treatments on increasing fruit juice content Bakry (2007) on Java orange and El-Shamma et al., (2013) on Valencia orange trees.

Table 6. Effect of foliar spray with yeast extract, sea weeds extract, promalin and humic acid on juice total soluble solids, total acidity and TSS /acidity content of Washington Navel orange fruits during 2019 and 2020 seasons.

\begin{tabular}{|c|c|c|c|c|c|c|}
\hline \multirow{2}{*}{$\begin{array}{l}\text { Parameters } \\
\text { Treatments }\end{array}$} & \multicolumn{2}{|c|}{ TSS (\%) } & \multicolumn{2}{|c|}{ Total acidity $(\%)$} & \multicolumn{2}{|c|}{ TSS /acidity ratio } \\
\hline & 2019 & 2020 & 2019 & 2020 & 2019 & 2020 \\
\hline T1- Control & $9.89 \mathrm{~g}$ & $10.10 \mathrm{~h}$ & $1.07 \mathrm{a}$ & $1.06 \mathrm{a}$ & $9.26 \mathrm{~d}$ & $9.63 \mathrm{f}$ \\
\hline T2- Yeast extract at $2.5 \mathrm{~m} / \mathrm{L}$ & $11.17 \mathrm{c}$ & $11.32 \mathrm{~d}$ & $1.04 \mathrm{a}$ & $1.03 \mathrm{ab}$ & $10.84 \mathrm{bc}$ & $11.00 \mathrm{~cd}$ \\
\hline T3-Yeast extract at $5.0 \mathrm{~m} / \mathrm{L}$ & $11.37 \mathrm{bc}$ & $11.65 \mathrm{c}$ & $1.04 \mathrm{a}$ & $1.03 \mathrm{ab}$ & $10.99 \mathrm{~b}$ & $11.38 \mathrm{bc}$ \\
\hline T4- Sea weeds extract at $0.2 \mathrm{~m} / \mathrm{L}$ & $10.25 \mathrm{ef}$ & $10.47 \mathrm{~g}$ & $1.05 \mathrm{a}$ & $1.05 \mathrm{a}$ & $9.81 \mathrm{~cd}$ & $10.05 \mathrm{def}$ \\
\hline T5- Sea weeds extract at $0.4 \mathrm{~m} / \mathrm{L}$ & $10.72 \mathrm{~d}$ & $11.09 \mathrm{e}$ & $1.04 \mathrm{a}$ & $1.04 \mathrm{a}$ & $10.30 \mathrm{bcd}$ & $10.70 \mathrm{cde}$ \\
\hline T6- Promalin at 100 ppm & $11.57 \mathrm{ab}$ & $11.89 \mathrm{~b}$ & $1.06 \mathrm{a}$ & $0.99 a b$ & $10.95 \mathrm{~b}$ & $12.00 \mathrm{ab}$ \\
\hline T7- Promalin at 200 ppm & $11.80 \mathrm{a}$ & $12.08 \mathrm{a}$ & $0.96 \mathrm{a}$ & $0.95 \mathrm{~b}$ & $12.30 \mathrm{a}$ & $12.78 \mathrm{a}$ \\
\hline T8- Humic acid at $3.0 \mathrm{~g} / \mathrm{L}$ & $10.53 \mathrm{de}$ & $10.79 \mathrm{f}$ & $1.05 \mathrm{a}$ & $1.04 \mathrm{a}$ & $10.10 \mathrm{bcd}$ & $10.38 \mathrm{def}$ \\
\hline T9- Humic acid at $6.0 \mathrm{~g} / \mathrm{L}$ & $10.10 \mathrm{fg}$ & $10.25 \mathrm{~h}$ & $1.07 \mathrm{a}$ & $1.05 \mathrm{a}$ & $9.52 \mathrm{~d}$ & 9.80 ef \\
\hline $\mathrm{T} 10=\mathrm{T} 2+\mathrm{T} 4+\mathrm{T} 6+\mathrm{T} 8$ & $11.58 \mathrm{ab}$ & $11.89 \mathrm{~b}$ & $1.06 \mathrm{a}$ & $0.99 \mathrm{ab}$ & $10.99 \mathrm{~b}$ & $12.05 \mathrm{ab}$ \\
\hline
\end{tabular}

Mean followed by the same letters within each column are not significantly different at 0.5 level.

Table 7. Effect of foliar spray with yeast extract, sea weeds extract, promalin and humic acid on juice total sugar (\%) and vitamin C content of Washington Navel orange fruits during 2019 and 2020 seasons.

\begin{tabular}{|c|c|c|c|c|}
\hline \multirow{2}{*}{$\begin{array}{l}\text { Parameters } \\
\text { Treatments }\end{array}$} & \multicolumn{2}{|c|}{ Fruit juice total sugar (\%) } & \multicolumn{2}{|c|}{ Vitamin $C$ content (mg/100 ml juice) } \\
\hline & 2019 & 2020 & 2019 & 2020 \\
\hline T1- Control & $6.38 \mathrm{~g}$ & $7.21 \mathrm{~h}$ & $36.64 \mathrm{~g}$ & $37.14 \mathrm{~h}$ \\
\hline T2- Yeast extract at $2.5 \mathrm{~m} / \mathrm{L}$ & $7.61 \mathrm{~d}$ & $8.20 \mathrm{~d}$ & $45.07 \mathrm{~d}$ & $48.44 \mathrm{~d}$ \\
\hline T3-Yeast extract at $5.0 \mathrm{~m} / \mathrm{L}$ & $7.77 \mathrm{c}$ & $8.33 \mathrm{c}$ & $49.57 \mathrm{c}$ & $52.54 \mathrm{c}$ \\
\hline T4- Sea weeds extract at $0.2 \mathrm{~m} / \mathrm{L}$ & $6.76 \mathrm{f}$ & $7.77 \mathrm{fg}$ & $38.80 \mathrm{f}$ & $40.37 \mathrm{~g}$ \\
\hline T5- Sea weeds extract at $0.4 \mathrm{~m} / \mathrm{L}$ & $7.06 \mathrm{e}$ & $7.99 \mathrm{e}$ & $42.50 \mathrm{e}$ & $45.60 \mathrm{e}$ \\
\hline T6- Promalin at 100 ppm & $8.43 \mathrm{~b}$ & $8.50 \mathrm{~b}$ & $53.04 \mathrm{~b}$ & $56.27 \mathrm{~b}$ \\
\hline T7- Promalin at 200 ppm & $8.70 \mathrm{a}$ & $8.74 \mathrm{a}$ & $57.64 \mathrm{a}$ & $60.17 \mathrm{a}$ \\
\hline T8- Humic acid at $3.0 \mathrm{~g} / \mathrm{L}$ & $6.92 \mathrm{ef}$ & $7.86 \mathrm{f}$ & $39.90 \mathrm{f}$ & $43.34 \mathrm{f}$ \\
\hline T9- Humic acid at $6.0 \mathrm{~g} / \mathrm{L}$ & $6.50 \mathrm{~g}$ & $7.68 \mathrm{~g}$ & $36.57 \mathrm{~g}$ & $38.54 \mathrm{~h}$ \\
\hline $\mathrm{T} 10=\mathrm{T} 2+\mathrm{T} 4+\mathrm{T} 6+\mathrm{T} 8$ & $8.50 \mathrm{~b}$ & $8.58 \mathrm{~b}$ & $53.07 \mathrm{~b}$ & $56.30 \mathrm{~b}$ \\
\hline
\end{tabular}

Mean followed by the same letters within each column are not significantly different at 0.5 level. 


\section{References}

Amer, S. A. (2004): Growth, green pods yield and seeds yield of common bean (Phaseolus vulgaris, L) as affected by active dry yeast, salicylic acid and their interaction. J. Agric. Sci. Mansoura. Univ., 29(3): 1407-1422.

Association of Official Analytical Chemists. A.O.A.C. (2000): Official method of analysis, 15 $\frac{\text { th }}{}$ Ed., Inc., USA.

Atawia, A. R. and El-Desouky, S. A. (1998): Trials for improving fruit set, yield and fruit quality of Washington Navel orange by application of some growth regulators and yeast extract as a natural source of phytohormones. Annals of Agricultural Science, Moshtohor; 35(3):1613- 1632.

Bakry, Kh. A. (2007): Response of Jafa orange trees to spray with yeast extract and promalin. Egypt. J. Appl. Sci.; 22 (10A): 195-210.

Burak, M.; Guardiola, J. L. and Quinlan, J. D. (1998): Effect of promalin on fruit shape and quality of Delicious apple cultivar. Acta Hort. No.463, 365369,4 ref.

Crouch, I. J. and Van-Staden, J. (1993): Evidence for the presence of plant growth regulators in commercial seaweed products. Plant Growth Regul.13: 2129.

Dhargalkar, V. K. and Pereira, N. (2005): Seaweed. Promising plant of the millennium. Science. 71(3-4): 60-66.

Duncan, D. B. (1955): Multiple range and multiple F tests. Biometrics, 11(1), 1-42.

El-Shamma, M. S.; Abd El-Motty, E. Z.; Zaied, N. S.; Maksoud, M. A. and Mansour, A. E. (2013): Efficiency of some organic fertilizers as safe resources on the performance of Valencia orange trees grown in newly reclaimed soils. World Applied Sciences Journal; 2013. 25(9):1263- 1269.

El-Shazly, S. M. and Mustafa, N. S. (2013):
Enhancement yield, fruit quality and nutritional status of Washington Navel orange trees by application of bio-stimulants. Journal of Applied Sciences Research; 2013. 9(8): 5030-5034.

Ibrahim,T. A.; Salem, S. E. and Guindy, L. F. (1994): The influence of gibberellic acid and promalin on yield and fruit quality of Washington Navel orange. Bull. Fac. of Agric. Cairo Univ., 45:3, 711-721, 17 ref.

Khan, W.; Rayirath, U. P.; Subramanian, S.; Jithesh, R. P.; Hodges, D. M.; Critchley, A. T.; Craigie, J. S.; Norrie, J. and Prithivira, B. (2009): Seaweed extracts as bio stimulants of plant growth and development", J. Plant Growth Regul., 28: 386399.

Mangan, R. L. and Thomas, D. B. (2014): Comparison of torula yeast and various grape juice products as attractants for Mexican fruit fly (Diptera: Tephritidae). Journal of Economic Entomology; 2014. 107(2):591-600.

Ministry of Agriculture \& Land Reclamation; Economic Affairs Sector, Egypt 2019.

Smith, F.; Cilles, A. M.; Hamilton, K. J. and Gedes, A. P. (1956): Colorimetric methods for determination of sugar and related substances. Annuals chem., 28: 350.

Snedecor, G. W. and Cochran, W. G. (1990): Statistical Methods, Eight Edition, Iowa State University Press.

Vaughan, D.; Malcom, R. E. and Ord, B. G. (1985): Influence of humic substances on biochemical processes in plants. In: Vaughan, D., Malcom, R.E. (Eds.), Soil Organic Matter and Biological Activit, Martinus Nijhoff/Junk W, Dordrecht, The Netherlands, pp: 77-108.

Westwood, M. N. (1978): Temperate zone pomology W. H. Freeman and Company. San Francisco.

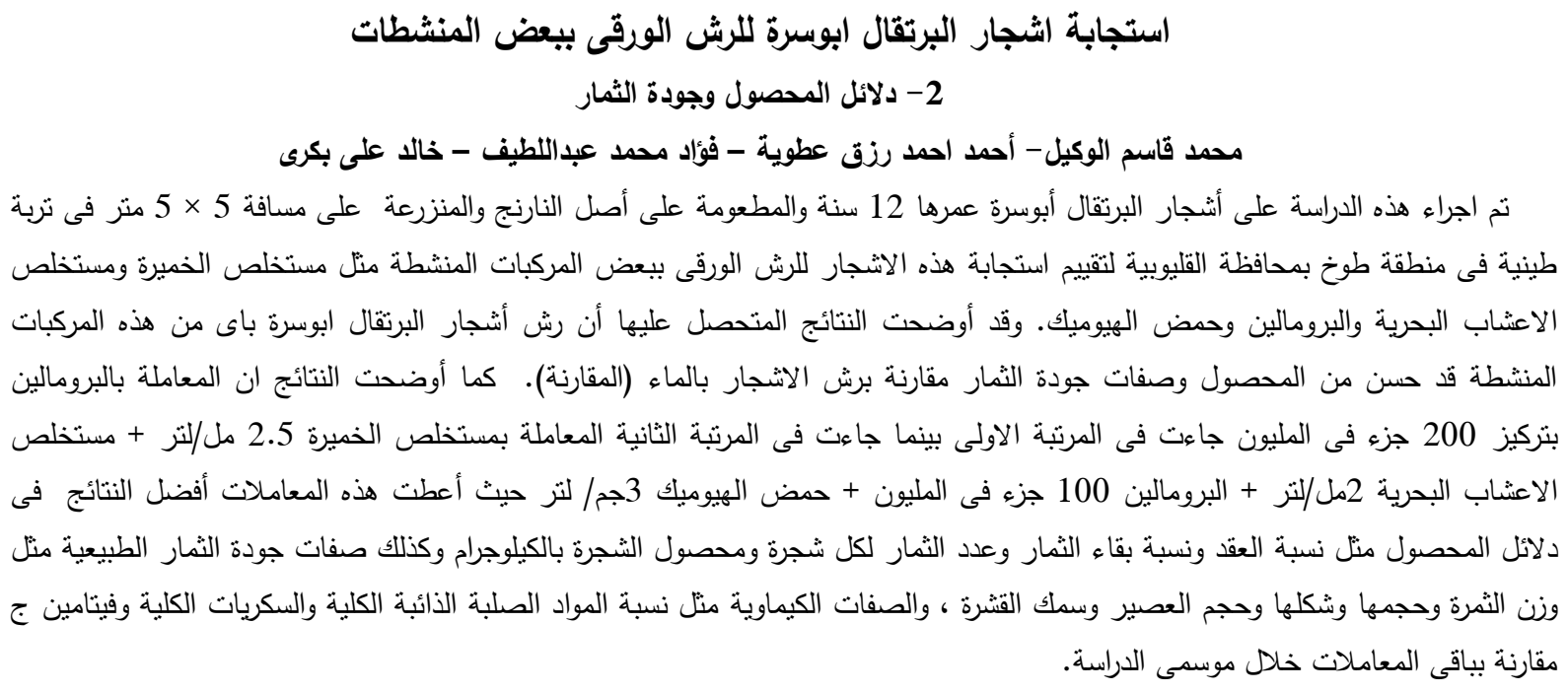

